\title{
Ratio of the Medial Hip Joint Space as a Predictor for Collapse of the Femoral Head in Non-traumatic Osteonecrosis: A Retrospective Study and Finite Element Analysis
}

Tianye Lin ( $\square$ lin_tye@163.com )

Guangzhou University of Chinese Medicine

\section{Peng Yang}

Guangzhou University of Chinese Medicine

Hongzhu Li

Guangzhou University of Chinese Medicine Jingli Xu

Guangzhou University of Chinese Medicine

\section{Binglang Xiong}

Guangzhou University of Chinese Medicine

\section{Zhenqiu Chen}

Guangzhou University of Chinese Medicine

Wei He

Guangzhou University of Chinese Medicine

\section{Qiushi Wei}

Guangzhou University of Chinese Medicine

Qing-Wen Zhang ( $\nabla$ zh_qwen@163.com )

Guangzhou University of Chinese Medicine https://orcid.org/0000-0002-5417-2970

\section{Research article}

Keywords: Osteonecrosis of the femoral head, biomechanics, medial space, collapse, finite element analysis

Posted Date: May 18th, 2020

DOl: https://doi.org/10.21203/rs.3.rs-27533/v1

License: (9) This work is licensed under a Creative Commons Attribution 4.0 International License. Read Full License 


\section{Abstract}

Background: There has been no indicators that can effectively predict femoral head collapse in ONFH so far. The aim of this study is to retrospectively analyze the first-visit medial space ratio of the hip joint to evaluate its efficacy in predicting ONFH-induced collapse and impacts on the mechanical environment of necrotic femoral head.

Methods: In this retrospective analysis for traditional Chinese medicine (TCM), non-traumatic osteonecrosis of femoral head (NONFH) patients from January 2010 to December 2016 were selected. The medial space ratio at their first visit and and collapse of the femoral head during the follow-up were recorded. Patients were divided into group A, B, C, D, and E according to the grading of the medial space ratio at the first visit. A total of 14 hip joint models with distinct medial space ratios were established. The maximum stress intensities of the cartilage, cortical bone and the necrotic area ( $\mathrm{N}$-unit area) of the femoral head in the models were quantitated using a finite element analysis.

Results: One hundred twenty-eight patients (142 hips) were included in this study. The average follow-up time was $5.4 \pm 1.5$ years. The Kaplan-Meier survival analysis showed that survival rates of the first-visit medial space ratios in group $C$ and $D$ were significantly higher (exceeding $45.76 \%$ ) than that in group $E$ (22.73\%). There was no significant difference in the survival rates between group $C$ and $D(P>0.05)$. The finite element analysis showed that in either the necrosis or non-necrosis group, the maximum stress in cartilage, cortical bone and the $\mathrm{N}$-unit area of the femoral head increased with the medial space ratio decreasing.

Conclusion: The medial space ratio shows clinical implications of predicting the collapse of NONFH except for assessing the relationship between the femoral head and the acetabulum. The stress concentrations of cartilage, cortical bone, and the necrotic area of the femoral head are enhanced with the medial space radio decreasing. Once the medial space ratio falls below 4 and continues to decline, the risk of necrotic collapse will become higher.

\section{Background}

Osteonecrosis of the femoral head (ONFH), a common hip disease as a result of trauma, steroid use, alcoholic abuse and other pathogenic factors, can bring about blood flow disorders in the femoral head and cell apoptosis in bone cells and bone marrow hematopoietic cells. ONFH is an increasing global health problem [1-3] as it is three times more prevalent in men than women, with the prevalence of bilateral ONFH found in up to $75 \%$ of the cases $[4,5]$. Moreover, undiagnosed and untreated ONFH can eventually progress to collapse of the femoral head [6-8]-the most critical pathological feature in ONFH, repair of ONFH and hip osteoarthritis-which may attribute to both biological and biomechanical factors [9]. Despite the size and shape of the necrotic area, the collapse is also strongly associated with the internal mechanical changes of the femoral head after necrosis. Therefore, an effective predictor for 
femoral head collapse is the prerequisite for a successful treatment of ONFH. Unfortunately, such indicators for the effective prediction of ONFH-induced collapse have not been found so far.

The medial hip joint space refers to the distance from the most medial margin of the femoral head to the pelvic teardrop. The medial space ratio is defined as the ratio of the medial hip joint space to the distance from the pelvic teardrop to the outermost margin of the acetabulum. Two studies $[10,11]$ report that the degree of hip dislocation can be evaluated by the medial hip space which exhibits clinical implications of judging joint dysplasia or dislocation. Yoshida et al. [12] report that in the development dysplasia of the hip, local stress concentration due to the reduction in the contact area of the hip joint results in asymmetric cartilage degeneration and the subsequent increases in joint contact pressure. Along with the size of the joint contact area that has the greatest influence on the contact stress, from a biomechanical perspective, changes in the hip joint contact area in ONFH may have an impact on the stress environment inside the femoral head. On account of the medial space ratio that reflects the contact area between the acetabulum and the femoral head, we hypothesize that there can be a clear correlation between the medial space ratio and femoral head collapse in $\mathrm{ONFH}$, which can predict the prognosis of ONFH.

In this study, we have reviewed the cases of non-traumatic ONFH admitted to our hospital, and have collected and dynamically observed the changes of the medial hip joint space on the X-ray. Survival analysis has been performed to compare the femoral head survival among groups with grade $y$ collapse as the end point. Furthermore, the finite element technology has been employed to explain the impacts of the medial space ratio on the internal stress environment of the ONFH from the perspective of biomechanics, and to improve the imaging evaluation system of ONFH for predicting the prognosis at an early stage. This study may shed new light on the early treatment of ONFH.

\section{Methods}

\section{Clinical research methods Research object}

This study retrospectively analyzed the non-traumatic, non-surgical ONFH patients admitted to the First Affiliated Hospital of Guangzhou University of Chinese Medicine from January 2010 to December 2016. The inclusion criteria were defined as (i) patients with complete imaging data and without collapse of the femoral head at the first visit, (ii) aged 19 to 60 years old, (iii) who reported no hip trauma and history of surgery. The exclusion criteria were as follows: the imaging data were incomplete during the follow-up, or available imaging data with poor quality (such as pelvic tilt). Patients with cardio-cerebral vascular diseases, nervous system diseases, severe illness, or rheumatoid arthritis were also excluded. This study had been approved by the ethical review board of The First Affiliated Hospital of Guangzhou University of Chinese Medicine (No: Y[2019]118). 


\section{Conservative Treatment}

All patients always accepted oral administrations with TCM Yuanshi Shengmai Chenggu tablet (6 tablets each time, 3 times per day, institutional approval number: Z20070828) and Fufang Shengmai Chenggu capsule (4 capsules each time, 3 times per day, institutional approval number: Z20071224). The two drugs were prepared by the First Affiliated Hospital of Guangzhou University of Traditional Chinese Medicine (Guangzhou, China). Along with the oral administration, muscle group exercises with emphasis on anterior flexor muscles, abductor muscles and adductor muscles and protective weight-bearing exercises were performed.

\section{Imaging evaluation of necrotic collapse}

Necrotic collapse was evaluated by comparing patients' X-ray radiographs simultaneously with the standard photographic grading of the hip joint in the quantitative collapse standard proposed by the Association Research Circulation Osseous (ARCO) in 1993 [13]. The 4 grades for necrotic collapse were defined as: grade $a$, no collapse; grade $\beta$, collapse of the femoral head of less than $2 \mathrm{~mm}$ or $15 \%$; grade $\gamma$, collapse of less than $4 \mathrm{~mm}$ or $30 \%$; and grade $\delta$, collapse of less than $4 \mathrm{~mm}$ or greater than $30 \%$.

\section{Definition of the medial space ratio}

According to the standard hip joint X-ray radiographs, a vertical tangent line "a" was drawn from the medial edge of the femoral head, and a vertical line "b" was drawn at the lowest point of the ipsilateral acetabular teardrop. The distance " $c$ " between the two lines a and b was the medial space of the hip joint. Subsequently, a vertical line " $d$ " at the outermost margin of the ipsilateral acetabulum was drawn, parallel to line $a$ and line $b$. The distance from line $b$ to line $d$ was distance "e", and the e/c ratio was defined as the medial space ratio (Fig. 1).

\section{Grouping according to the first-visit medial space ratio}

The medial space ratio was classified and assigned based on the data at the first visit (Table 1). Because the size of the medial space at the initial diagnosis was smaller than that at the end point in group $A(n=$ $0), B(n=3)$, and $F(n=0)$, the indice was excluded. This analysis mainly used data from the first-visit medial space ratio in group $C(n=36), D(n=71)$, and $E(n=32)$ as variables.

Table 1

Grouping according to the first-visit medial space ratio

\begin{tabular}{|lllllll|}
\hline Grouping & F & E & D & C & B & A \\
\hline $\begin{array}{l}\text { First visit medial space } \\
\text { ratio }\end{array}$ & $2<=\mathrm{F}<$ & $3<=\mathrm{E}<$ & $4<=\mathrm{D}<$ & $5<=\mathrm{C}<$ & $6<=\mathrm{B}<$ & $\begin{array}{l}7<=\mathrm{A}< \\
8\end{array}$ \\
\hline
\end{tabular}


The Kaplan-Meier survival analysis of the first-visit medial space ratio was performed with grade $Y$ collapse of the femoral head as the endpoint event. The above data were imported into SPSS version 22.0 statistical analysis software, and a $P$ value of $<0.05$ was considered statistically significant.

\section{Finite element experiments}

\section{Establishment of 3D models of the total hip joint}

A 30-year-old healthy woman was excluded from our analysis due to the history of hip and systemic diseases. DICOM data obtained from computerized tomography(CT)scans were imported into the Mimics 16.0 system (Fig. 2A,B). After threshold segmentation, approximate contours of the cortical bone in the femur and patella were obtained, and then repaired by region growth, skin editing, and cavity filling. After the surface was smoothed, Those models were exported as a stl file format and imported into the Geomagic-Studio 11 system to mesh and fit the surface to obtain a stp-format cortical bone model. The above steps were repeated to obtain a cancellous bone model, and then it was imported in the Solidworks 2014 software for a 3D model of the femoral and patella bone structures. Subsequently, the thickness and contour of the cartilage in the bony 3D model were defined based on MRI images after registration and fusion using the Solidworks 2014 software system, and a 3D surface was constructed based on the contour curve. Finally, independent 3D models of femoral head cartilage and acetabular cartilage were reconstructed (Fig. 2C,D,E).

\section{Establishment of necrotic zone models}

The JIC type $\mathrm{C} 1 \mathrm{ONFH}$ was used as a model reference to establish a necrotic zone model. A circle $a$ was drew around the center of the femoral head using the Solidworks software (Fig. 3A). Then the circle $a$ was stretched and the femoral head was cut. Finally, the JIC type C1 ONFH model including the necrotic area was established (Fig. 3B).

\section{Establishment of multiple hip joint models based on graded medial space ratios}

Based on data from clinical research, the medial space ratio and CE angle were calculated. The hip joint models were divided into 7 groups with a reduction of every 0.3 in the medial space ratio. The origin control was defined as the starting coordinate point in Solidworks software environment and the lateral direction of the hip joint as the positive direction of the $X$ axis to move the model of the femur and other components to the outside. Seven models $a, \beta, \gamma, \delta, \varepsilon, \zeta$, and $\eta$ (Fig. 4) were established according to the medial space ratios (Table 2). Furthermore, 14 models were constructed based on the presence or absence of necrotic areas in the 7 models: non-necrotic model $a, \beta, \gamma, \delta, \varepsilon, \zeta$, and $\eta$, and necrotic model $a$, $\beta, \gamma, \delta, \varepsilon, \zeta$, and $\eta$. 


\section{Establishment of different medial space hip joint models}

Table 2

Setting of the medial space ratio of each model

\begin{tabular}{|lllll|}
\hline & $\begin{array}{l}\text { medial } \\
\text { space }\end{array}$ & $\begin{array}{l}\text { medial space } \\
\text { ratio }\end{array}$ & $\begin{array}{l}\text { CE } \\
\text { angle }\end{array}$ & $\begin{array}{l}\text { Distance from teardrop to outer edge of } \\
\text { acetabulum }\end{array}$ \\
\hline $\begin{array}{l}\text { a } \\
\text { model }\end{array}$ & $1.11 \mathrm{~cm}$ & 4.74 & 39.2 & 5.23 \\
\hline $\begin{array}{l}\beta \\
\text { model }\end{array}$ & $1.17 \mathrm{~cm}$ & 4.44 & 35.1 & 5.23 \\
\hline $\begin{array}{l}\text { model } \\
\text { mode }\end{array}$ & $1.26 \mathrm{~cm}$ & 4.14 & 31.4 & 5.23 \\
\hline $\begin{array}{l}\delta \\
\text { model }\end{array}$ & $1.36 \mathrm{~cm}$ & 3.84 & 28.2 & 5.23 \\
\hline $\begin{array}{l}\varepsilon \\
\text { model }\end{array}$ & $1.47 \mathrm{~cm}$ & 3.54 & 25.4 & 5.23 \\
\hline $\begin{array}{l}\zeta \\
\text { model }\end{array}$ & $1.61 \mathrm{~cm}$ & 3.24 & 22.5 & 5.23 \\
\hline $\begin{array}{l}\eta \\
\text { model }\end{array}$ & $1.77 \mathrm{~cm}$ & 2.94 & 20.0 & 5.23 \\
\hline
\end{tabular}

\section{Analysis of settings of the finite element model}

\section{Meshing and assignment of material properties}

The assembled 3D model was imported into the Abaqus 6.14 system and meshed to establish a finite element mesh model of the hip joint. The model of the hip joint contains 61,044 nodes and $3,72,708$ elements. As with details reported by Grecu $D^{[14]}$, cortical bone, cancellous bone and cartilage were made by isotropic, continuous and uniform elastic materials (Table 3 ).

Table 3

Assignment of material properties

\begin{tabular}{|lll|}
\hline Material & Elastic Modulus(MPa) & Poisson's ratio \\
\hline Cortical bone & 15100 & 0.3 \\
\hline Cancellous bone & 4457 & 0.22 \\
Cartilage & 10.5 & 0.45 \\
\hline Necrotic tissue & 124.6 & 0.152 \\
\hline
\end{tabular}


The 6 degrees of freedom that constrained the pelvic joint and the pubic bone were zero. The articular surfaces were set in a frictionless contact relationship. According to Sverdlova et al. [15], 6 muscle forces were loaded on the coupling surfaces corresponding to the attachment point with the axial connection force (Table 4). Based on the settings reported by Brown et al. [16], the joint force was 1.6 times the body weight and loaded on the rigid body of the distal femur. (Fig. 5). The relationship between the femoral cartilage and the acetabular cartilage was set as friction, and the coefficient of friction was 0.2 . The relationship of other model parts were set as the binding relation. The results of convergence test for the element size showed that the errors were below $10 \%$.

Table 4

Muscle load settings

\begin{tabular}{|lllllll|}
\hline Muscle & $\begin{array}{l}\text { Adductor } \\
\text { longus }\end{array}$ & Pestineus & $\begin{array}{l}\text { Gluteus } \\
\text { maximus }\end{array}$ & $\begin{array}{l}\text { Gluteus } \\
\text { medius }\end{array}$ & $\begin{array}{l}\text { Gluteus } \\
\text { minimus }\end{array}$ & Piriformis \\
\hline Myodynamia(N) & 560 & 500 & 550 & 700 & 300 & 500 \\
\hline
\end{tabular}

\section{Experimental indicators}

The VM stress distribution in the cartilage and cortical bone in the femoral head were observed and recorded. In order to study the stress distribution of the necrotic area in necrotic models, the unit area of the overlapped necrotic tissue in necrotic and non-necrotic models was defined as the $\mathrm{N}$-unit area. The VM stress distribution of the $\mathrm{N}$-unit area was also observed and recorded.

\section{Results}

\section{Clinical Research Results}

\section{General information}

A total of 128 patients (92 males and 36 females) with 142 hips were included in this study, 14 of whom had bilateral ONFH (Table 5). Their age ranged from 19 to 60 years, with an average age of $43.65 \pm$ 12.48 years. All included patients were diagnosed as ONFH by radiologists in our department and orthopedic experts at our hospital according to their eligible radiographs, and the initial grading of necrotic collapse was grade $a$. The follow-up period lasted from 3 to 7 years, with an average duration of $5.4 \pm 1.5$ years. 
Table 5

Clinical characteristics of patients with non-traumatic

ONFH

\begin{tabular}{|lll|}
\hline Items & Category & Number of hips (percent) \\
\hline \multirow{2}{*}{ gender } & Male & $82(63.8 \%)$ \\
\cline { 2 - 3 } & Female & $46(36.2 \%)$ \\
Cause & Left & $76(53.5 \%)$ \\
\cline { 2 - 3 } & Right & $66(46.5 \%)$ \\
& Hormone & $63(44.4 \%)$ \\
\cline { 2 - 3 } JIC typing & Alcohol & $48(33.8 \%)$ \\
& Idiopathic & $31(21.8 \%)$ \\
& B & $4(2.8 \%)$ \\
& C1 & $72(50.7 \%)$ \\
& C2 & $17(12 \%)$ \\
\hline
\end{tabular}

\section{Effect of medial space ratio at first visit on femoral head collapse}

According to the statistical results, the P-value of the log-rank test used to compare the first-visit medial space ratio among groups was less than 0.05 . Therefore, it was considered that within the $95 \%$ confidence interval the differences in survival rates of the femoral heads were measured using the firstvisit medial space ratios from C, D, and E groups as variables (Table 6). According to the survival curve, the grade $y$ collapse of the femoral head was the end point of this survival analysis. The survival rate of the first-visit medial space ratio of group $E$ was significantly different from those of group $C$ and $D$, featuring a lower rate in group $E$ than the other two groups. There was no significant difference in survival rate between group $C$ and $D$. The survival rates of the first-visit medial space ratio in group $C$ and $D$ exceeded $45.76 \%$, while the final survival rate in group E was $22.73 \%$, which was far lower than those in group $C$ and $D$ (Fig. 6). Clinical experiments showed that patients with higher medial space ratios (Fig. 7) had a better prognosis than those with lower medial space ratios (Fig. 8). The femoral heads in patients with lower medial space ratios were more likely to collapse and progress to hip arthritis, which required total hip replacement. 
Table 6

Log-rank (Mantel-Cox) test

results of the medial space ratio

\begin{tabular}{|lc|}
\hline \multicolumn{2}{|l|}{ Log-rank (Mantel-Cox) test } \\
\hline Chi square & 13.579 \\
\hline df & 2 \\
\hline P value & 0.001 \\
\hline
\end{tabular}

\section{Experimental research results}

\section{Distribution of the maximum VM stress in femoral head cartilage}

The maximum VM stress distribution was found at the top of the femoral head cartilage in each model group, and stress intensity gradually decreased radially around the maximum stress area. With decreases in the medial space ratio, the maximum stress distribution in femoral head cartilage moved inward and downward obviously (Fig. 9). As shown in Fig. 10A, VMstress intensity increased as the medial space ratio decreased, and such a variation was obvious at the stage when the medial space ratio fell below 4 .

\section{Distribution of the maximum VM stress in the cortical bone of the femoral head}

In both the necrosis and non-necrosis groups, the distribution of the maximum VM stress on the cortical bone surface of the femoral head was located on the anterolateral side of the top of the femoral head. In the non-necrosis group, stresses obviously concentrated at the top of the femoral head coincident with the position of the weight-bearing area, and gradually decreased around the center of the maximum VM stress area. A color difference appeared at the edge of the weight-bearing area. As the medial space ratio decreased, the stress concentration band at the top of the femoral head moved inward. In the necrosis group, the maximum stress intensity obviously concentrated at the top of the femoral head. Compared with the non-necrosis group, the stress intensity at the top of the femoral head was much higher than that in the remaining area in the medial space (Fig. 11). The magnitude of changes in the maximum stress in the necrosis group was greater than that in the non-necrosis group, indicating that the maximum stress on the cortical bone surface where ONFH commonly occured was more concentrated as the medial space narrowed. As the medial space ratio declined, the maximum stress of the cortical bone of the femoral head increased (Fig. 10B).

\section{Distribution of the maximum VM stress in the $\mathrm{N}$-unit area}


The maximum VM stress distribution in the N-unit area significantly varied between the necrosis group and the non-necrosis group. In the non-necrosis group, VM stresses concentrated in the N-unit area near the center of the femoral head, decreasing from top to bottom. In the necrosis group, stress distribution was pinpointed at the junction between the $\mathrm{N}$-unit area and normal tissues, and the maximum stress intensity appeared at the top outer edge. The maximum stress concentration in the N-unit region corresponded to the femoral head weight-bearing region, and the stress intensity near the center of the femoral head at the bottom of the $\mathrm{N}$-unit region was lower than that in the surrounding areas. The maximum VM stress of the $\mathrm{N}$-unit area in the non-necrosis group $(0.635 \pm 0.0077 \mathrm{MPa})$ was higher than that in the necrosis group $(0.444 \pm 0.0186 \mathrm{MPa})$ (Fig. 12).

In both the necrosis and non-necrosis groups, the maximum stress intensity in the N-unit area increased with the the medial space ratio decreasing, and the maximum stress in the necrotic area changed more significantly (Fig. 10C). Due to distinct tissue properties between the necrotic area and the surrounding areas, the elastic modulus of the necrotic area decreased, and the stress shielding effect of the surrounding normal tissues produced stress concentration particularly in the cortical bone. The formation of the material interface made it easier to generate stress concentration, which increased with the occurrence of lateral dislocation of the femoral head.

\section{Discussion}

In this study, the clinical research section has retrospectively studied cases of NONFH and analyzed dynamic changes in the medial space ratio of the hip joint on the X-ray. In the finite element analysis, 14 hip joint models with different medial hip joint spaces have been established for comparing stress distribution in the femoral head and the necrotic area between the necrosis and non-necrosis groups. This study has found that the medial space ratio has the clinical implication of predicting the collapse of the femoral head in NONFH patients except for assessing the distance from the femoral head to the acetabulum.

Clinical observations of ONFH patients show that the wider the medial hip joint space (or the smaller the hip medial space ratio) is, the more likely collapse of the femoral head is to happen. Anderson et al. [17] have retrospectively studied changes in the medial space of the hip joint in patients diagnosed as LeggCalve-Perthes disease in 1970. After analyzing imaging data from 50 patients with Legg-Calve-Perthes disease, they report that more than $50 \%$ of the cases present widened medial spaces, which can be interpreted as a manifestation of joint instability. Moreover, Gershuni et al. [18] also analyze the medial hip joint space in patients with Legg-Calve-Perthes disease. Jaramillo et al. [19] conduct a prospective study of 12 cases of Legg-Calve-Perthes disease and measure changes in the medial hip joint spaces using MRI. They grade the joint instability as normal, mild, moderate, and severe according to the medial spaces of the patients. Although they merely achieve nonsignificant results, they believe that there is an underlying association between the widened medial space and NONFH. The medial space width in normal individuals often remains stable under certain stress stimuli, and the changes in the joint space somewhat matters the variations in the hip joint [17]. In our present study, the Kapian-Meier survival 
analysis is performed to compare the first-visit medial space ratio with grade $y$ collapse of the femoral head as the endpoint event. We find that the lower the medial space ratio is, the more likely collapse of the femoral head is to happen and the lower the survival rate of the femoral head will be. This indicates that the medial space ratio is an indicator for predicting femoral head collapse in $\mathrm{ONFH}$.

Physiologically, the medial space reflects how well the acetabulum covers the femoral head. Accordingly, the relationship between the acetabulum and the femoral head can be speculated through variations in the medial space. Taketa $\mathrm{M}$ et al. [20] report that acetabular dysplasia may be an exacerbating factor of ONFH in 2003, but no further imaging measurements or analyses of acetabular morphology are performed. Besides, they report that the incidence of ONFH is higher in patients with hip dysplasia. When the center edge angle is small or the sharp angle is big, the incidence of ONFH rises. Russell ME et al. [21] compare the stress of the hip joint between normal controls and patients with hip dysplasia using a finite element analysis. They find that increases in the hip contact pressure of the patients are 1-2 times higher than the increases in normal controls. The bearing area is significantly reduced in the patients. A reduction in acetabular coverage to the femoral head causes the significant increase in hip contact pressure per unit area of the femoral head. And long-term weight-bearing squeeze can prolong the course of ONFH and accelerate collapse of the femoral head. Some researchers [22] report that the pressure in the hip joint capsules with reduced acetabular coverage is significantly higher than that of normal hip joints. The widening of the medial space leads to a reduction in the coverage of the femoral head, directly increasing the stress intensity in the articular cavity of the hip joint. And the resulting insufficient blood supply to the femoral head and impaired venous return can, in turn, affect the repair of ONFH. Similarly, some studies demonstrate that less acetabular coverage can increase intracapsular pressure, and excessive weight bearing suffered by the hip joint can contribute to the development of ONFH [23-26]. Junfeng Zeng et al. [27] find that less acetabular coverage may be associated with the development of ONFH in East Asian population, which is also a factor affecting the repair of ONFH. Thomas et al. [28] retrospectively investigate ONFH patients who accept free vascularized fibular grafting, and find that patients with less acetabular coverage $\left(\mathrm{a} C \mathrm{CE}\right.$ angle $\left.<30^{\circ}\right)$ present a higher prevalence of failure of repair than patients with a $\mathrm{CE}$ angle $>30^{\circ}$. In this study, we reconstruct hip joint models with different medial space ratios and conduct finite element experiments. The stress intensity in the cartilage, cortical bone and necrotic area of the femoral head increases with the medial space ratio decreasing. Moreover, comparing the model results of the necrosis group with the non-necrosis group, we also find that influences of changes in the medial space on the necrotic models are greater than those on non-necrotic ones. Mismatched acetabulum and femoral head caused by widened medial space may have more severe effects on patients with NONFH, which is why the risk of collapse and the degree of collapse in necrotic patients with femoral head dislocation are higher than than non-necrotic ones in our clinical observation. Therefore, we believe that the hip joint instability induced by abnormal stress concentrations in the femoral head can be an important cause of collapse. The widening of the medial space and the mismatch between the acetabulum and femoral head exacerbate the instability of stress distribution in the femoral head. Ultimately, the risk of collapse rises, consistent with the results of clinical studies. Therefore, we preliminarily confirm that the risk of collapse of the femoral head in ONFH patients 
becomes higher when the medial space ratio is less than 4 , so the medial space ratio is a promising indicator for predicting the collapse of the femoral head.

In the present study, we innovatively explore the relationship between the medial space of the hip joint and collapse of the femoral head. Furthermore, 3D finite element technology is employed to analyze the effects of changes in the medial space on the mechanical environment of the necrotic femoral head, which provide a new indicator for predicting the prognosis of NONFH. The limitation of this study is that the clinical research is a retrospective study, with limited cases and a relatively short period of follow-up. In addition, there is a certain disparity between a hip joint model in the finite element experiment and a real hip joint. The setting of material properties of single necrotic tissue can not fully simulate the complex pathological changes in reality. Therefore, more randomized clinical and biomechanical trials are still needed to verify the value of the medial space in predicting collapse of the femoral head.

\section{Conclusion}

The first-visit medial space ratio of the hip joint is important in predicting collapse of the necrotic femoral head because the medial space ratio is more objective and accurate in assessing the relationship between the femoral head and acetabulum. The stress intensity in the cartilage, cortical bone and necrotic areas of the femoral head increases with the medial space ratio decreasing. Therefore, the change in the medial space is a necrosis- and collapse-specific risk factor. And our study for the first time demonstrates that the risk of necrotic collapse rises when the medial space ratio falls below 4 .

\section{Abbreviations}

ONFH:Osteonecrosis of the femoral head

NONFH:Non-traumatic Osteonecrosis of the femoral head

TCM:Traditional Chinese medicine

3D:Three-dimensional

VM:Von Mises

CT:Computerized tomography

\section{Declarations}

\section{Availability of data and materials}

The datasets used in the current study are not publicly available due to privacy protection but are available from the corresponding author on reasonable request. 


\section{Acknowledgement}

Grateful acknowledgement is made to my supervisor Professor He Wei who gave me considerable help by means of suggestion, comments and criticism. In addition, I deeply appreciate the contribution to this thesis made in various ways by my friends and classmates.

\section{Funding}

Publication charges for this article were funded by Natural Science Foundation of China (81873327), Natural Science Foundation of Guangdong (2015A030313353), Excellent Doctoral Dissertation Incubation Grant of First Clinical School of Guangzhou University of Chinese Medicine.

\section{Author Contributions}

L.T.Y was responsible for the design and implementation of the study presented. L.T.Y and Y.P were responsible for the development of the finite-element models, C.Z.Q,X.B.L,H.W,X.J.L.and L.H.Z. conducted the experiments and were responsible for the acquisition of the data. L.T.Y and Y.P prepared the initial draft of the manuscript. Z.Q.W.and W.Q.S gave critical feedback during the study and critically revised the submitted manuscript for important ntellectual content. All authors have read and approved the final manuscript to be submitted.

\section{Ethical approval}

This study was conducted in agreement with the Declaration of Helsinki and its later amendments or comparable ethical standards and had been approved by the ethics board of The First Affiliated Hospital of Guangzhou University of Chinese Medicine (No: Y[2019]118).

\section{Consent for publication}

Not applicable.

\section{Competing interests}

The authors declare no competing interests.

\section{References}

1. Mont MA, Cherian JJ, Sierra RJ, Jones LC, Lieberman JR. Nontraumatic Osteonecrosis of the Femoral Head: Where Do We Stand Today? A Ten-Year Update. J Bone Joint Surg Am. 
2015;97:1604-27.

2. Kang JS, Park S, Song JH, Jung YY, Cho MR, Rhyu KH. Prevalence of osteonecrosis of the femoral head: a nationwide epidemiologic analysis in Korea. J Arthroplasty. 2009;24:1178-83.

3. Cui L, Zhuang Q, Lin J, Jin J, Zhang K, Cao L, et al. Multicentric epidemiologic study on six thousand three hundred and ninety five cases of femoral head osteonecrosis in China. Int Orthop. 2016;40:267-76.

4. Calori GM, Mazza E, Colombo A, Mazzola S, Colombo M. Core decompression and biotechnologies in the treatment of avascular necrosis of the femoral head. EFORT Open Rev. 2017;2:41-50.

5. Tabatabaee RM, Saberi S, Parvizi J, Mortazavi SM, Farzan M. Combining Concentrated Autologous Bone Marrow Stem Cells Injection With Core Decompression Improves Outcome for Patients with Early-Stage Osteonecrosis of the Femoral Head: A Comparative Study. J Arthroplasty. 2015;30:11-5.

6. Piuzzi NS, Chahla J, Schrock JB, LaPrade RF, Pascual-Garrido C, Mont MA, et al. Evidence for the Use of Cell-Based Therapy for the Treatment of Osteonecrosis of the Femoral Head: A Systematic Review of the Literature. J Arthroplasty. 2017;32:1698-708.

7. ohanty M, Singh SP, Kundangar KA, Shankar R. V. Management of nontraumatic avascular necrosis of the femoral head-a comparative analysis of the outcome of multiple small diameter drilling and core decompression with fibular grafting. Musculoskelet Surg. 2017;101:59-66.

8. Mont MA, Zywiel MG, Marker DR, McGrath MS, Delanois RE. The natural history of untreated asymptomatic osteonecrosis of the femoral head: a systematic literature review. J Bone Joint Surg Am. 2010;92:2165-70.

9. Nishii T, Sugano N, Ohzono K, Sakai T, Haraguchi K, Yoshikawa H. Progression and cessation of collapse in osteonecrosis of the femoral head. Clin Orthop Relat Res 2002: 149-57.

10. Offierski CM. Traumatic dislocation of the hip in children. J Bone Joint Surg Br. 1981;63-B:194-7.

11. Lequesne M, Malghem J, Dion E. The normal hip joint space: variations in width, shape, and architecture on 223 pelvic radiographs. Ann Rheum Dis. 2004;63:1145-51.

12. Yoshida H, Faust A, Wilckens J, Kitagawa M, Fetto J, Chao EY. Three-dimensional dynamic hip contact area and pressure distribution during activities of daily living. J Biomech. 2006;39:19962004.

13. Gardeniers JW. M. A new international classification of osteonecrosis of the ARCO committee on terminology and classification. J Jpn Orthop Assoc. 1992;66:18-9.

14. Grecu D, Pucalev I, Negru M, Tarnita DN, lonovici N, Dita R. Numerical simulations of the 3D virtual model of the human hip joint, using finite element method. Rom J Morphol Embryol. 2010;51:151-5.

15. Sverdlova NS, Witzel U. Principles of determination and verification of muscle forces in the human musculoskeletal system: Muscle forces to minimise bending stress. J Biomech. 2010;43:387-96.

16. Brown TD, Pedersen DR, Baker KJ, Brand RA. Mechanical consequences of core drilling and bonegrafting on osteonecrosis of the femoral head. J Bone Joint Surg Am. 1993;75:1358-67. 
17. Anderson J, Stewart AM. The significance of the magnitude of the medial hip joint space. $\mathrm{Br} \mathrm{J}$ Radiol. 1970;43:238-9.

18. Gershuni DH, Axer A, Hendel D. Arthrographic findings in Legg-Calve-Perthes disease and transient synovitis of the hip. J Bone Joint Surg Am. 1978;60:457-64.

19. Jaramillo D, Galen TA, Winalski CS, DiCanzio J, Zurakowski D, Mulkern RV, et al. Legg-Calve-Perthes disease: MR imaging evaluation during manual positioning of the hip-comparison with conventional arthrography. Radiology. 1999;212:519-25.

20. Taketa M, Fujii T, Kubota H, Nakashima Y, Noguchi Y, Iwamoto Y. Correlation between center-edge angle and acetabulum-head index in developmental dysplasia of the hip with avascular necrosis of the femoral head. J Pediatr Orthop B. 2003;12:215-8.

21. Russell ME, Shivanna KH, Grosland NM, Pedersen DR. Cartilage contact pressure elevations in dysplastic hips: a chronic overload model. J Orthop Surg Res. 2006;1:6.

22. Wingstrand $\mathrm{H}$, Wingstrand $A$. Biomechanics of the hip joint capsule -- a mathematical model and clinical implications. Clin Biomech (Bristol Avon). 1997;12:273-80.

23. Mihara K, Hirano T. Standing is a causative factor in osteonecrosis of the femoral head in growing rats. J Pediatr Orthop. 1998;18:665-9.

24. Hadley NA, Brown TD, Weinstein SL. The effects of contact pressure elevations and aseptic necrosis on the long-term outcome of congenital hip dislocation. J Orthop Res. 1990;8:504-13.

25. Wingstrand $\mathrm{H}$. Intracapsular pressure in congenital dislocation of the hip. J Pediatr Orthop B. 1997;6:245-7.

26. Xie J, Naito M, Maeyama A. Intracapsular pressure and interleukin-1 beta cytokine in hips with acetabular dysplasia. Acta Orthop. 2010;81:189-92.

27. Zeng J, Zeng Y, Wu Y, Liu Y, Xie H, Shen B. Acetabular Anatomical Parameters in Patients With Idiopathic Osteonecrosis of the Femoral Head. J Arthroplasty. 2020;35:331-4.

28. Roush TF, Olson SA, Pietrobon R, Braga L, Urbaniak JR. Influence of acetabular coverage on hip survival after free vascularized fibular grafting for femoral head osteonecrosis. J Bone Joint Surg Am. 2006;88:2152-8.

\section{Figures}




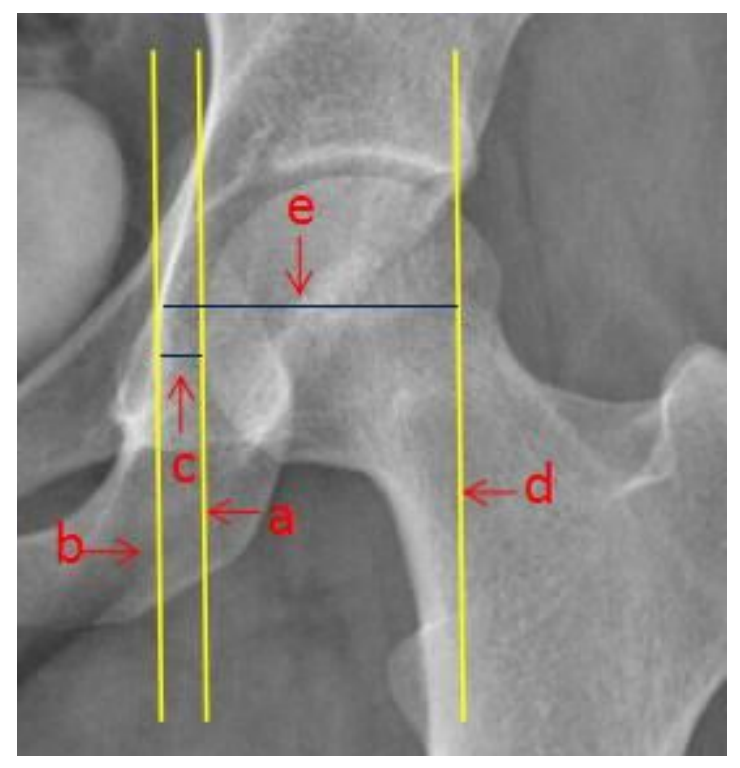

\section{Figure 1}

Imaging evaluation of the medial space ratio (defined by the e/c ratio)

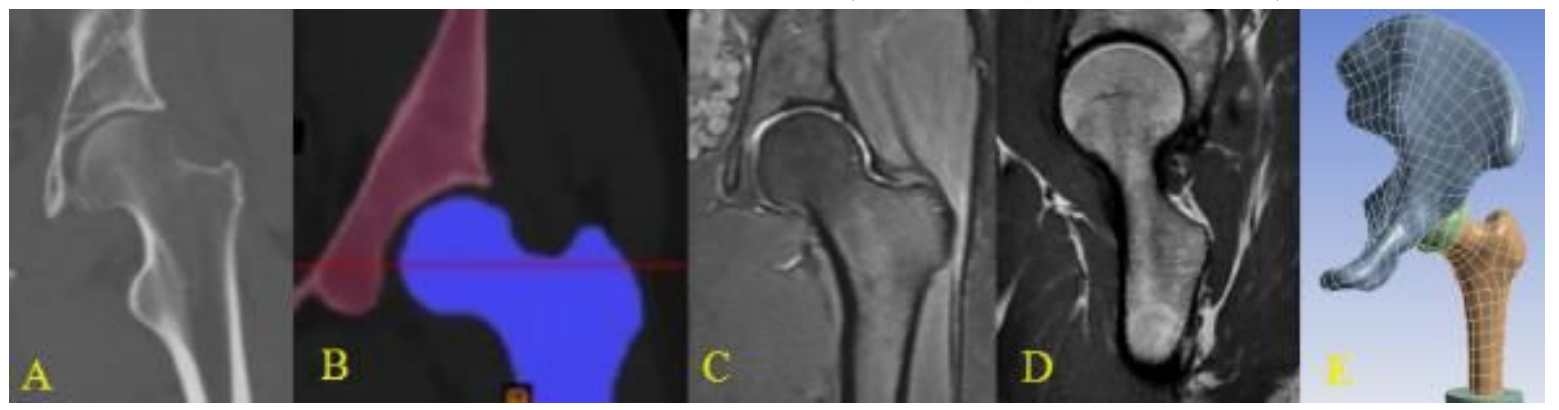

Figure 2

Construction of 3D finite element models of the hip joint based on CT (A, B) and MRI (C, D) imaging data 


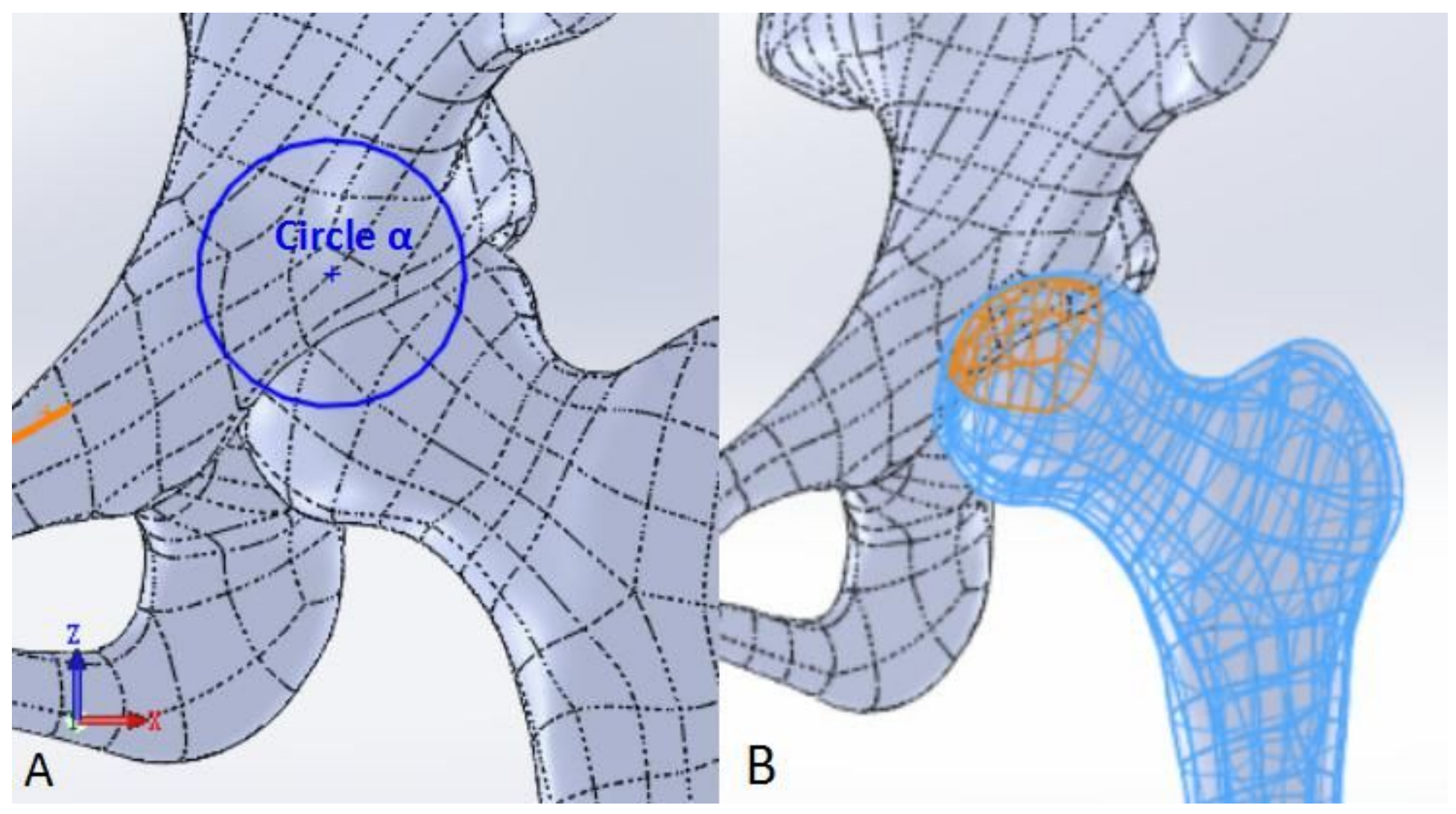

Figure 3

Establishment of a necrotic zone model. A: Coronal plane construction of the JIC type $\mathrm{C} 1$ sketch circle a. B: A JIC type C1 necrotic femoral head model was constructed.

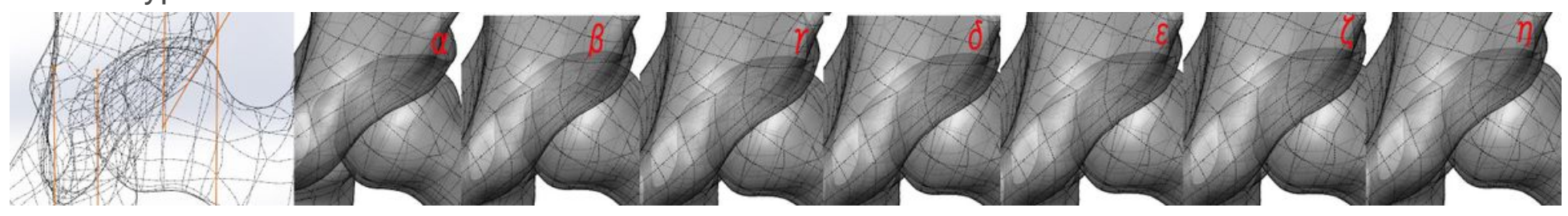

Figure 4

Building 7 models based on different medial space ratios 


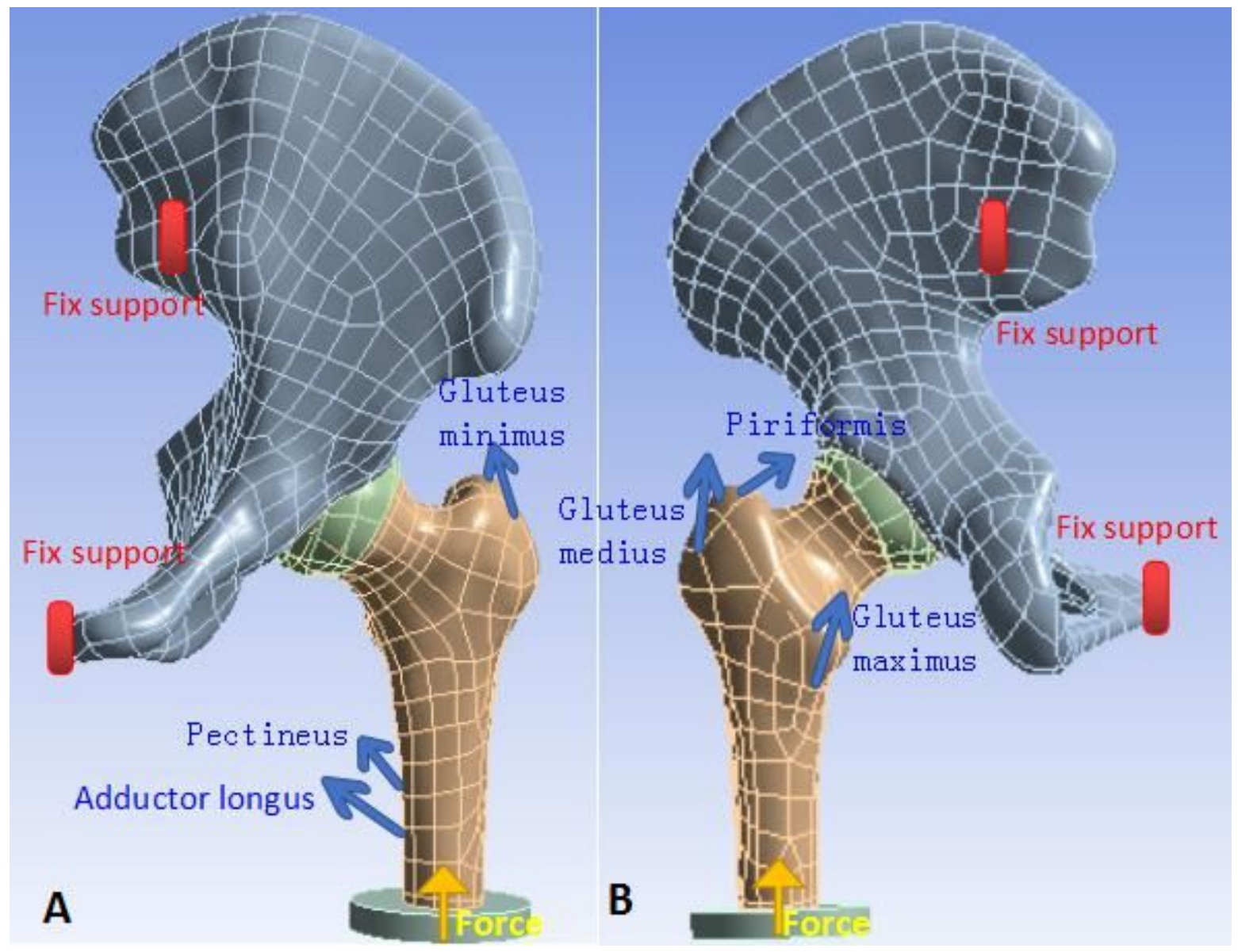

\section{Figure 5}

Front $(A)$ and back(B) views of a schematic diagram of a three-dimensional finite element model of the muscles around the hip joint, and the diagram of boundary conditions and loading force settings. 


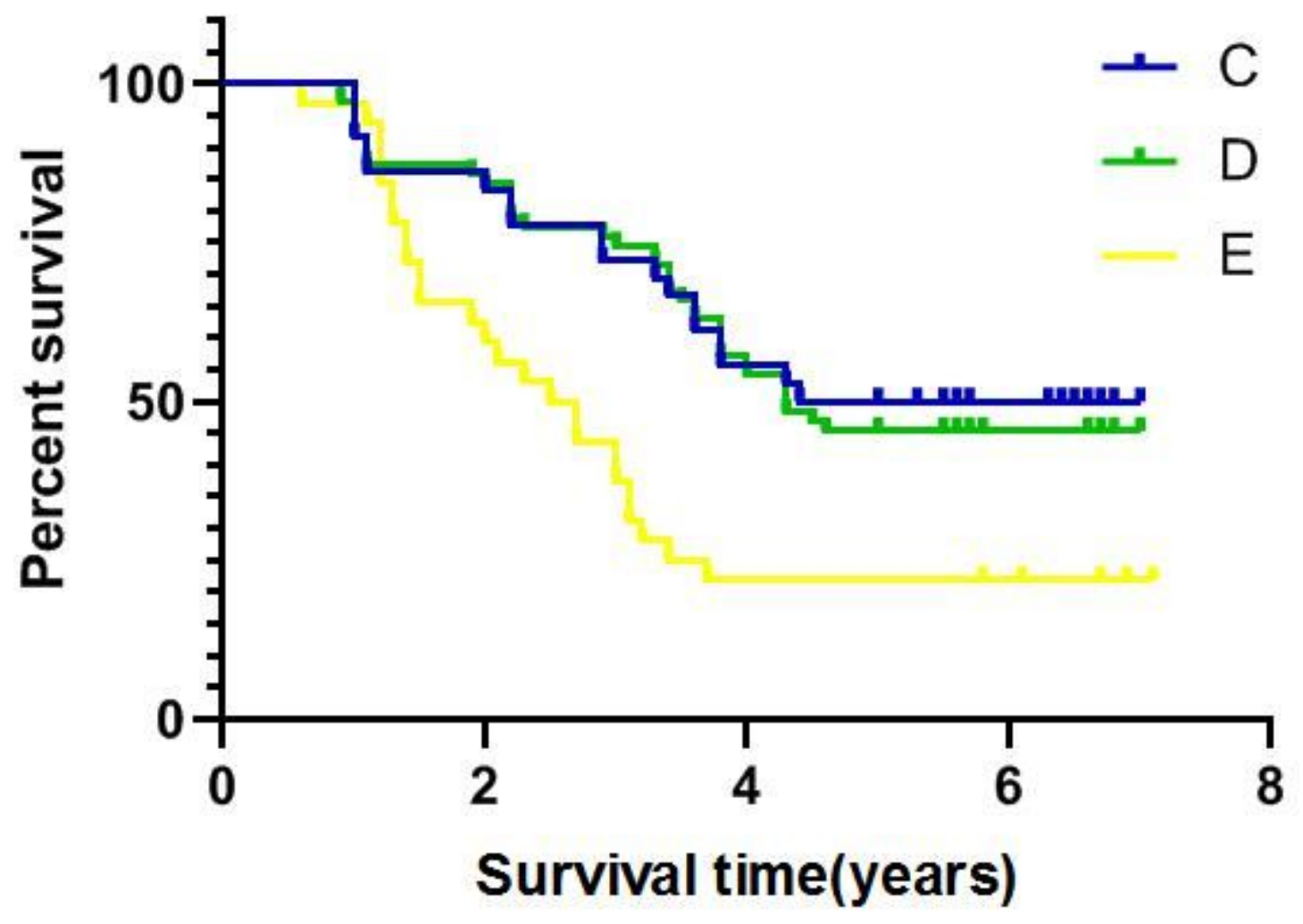

Figure 6

Survival rates of the femoral head using the medial space ratio as the variable and grade $y$ collapse as the end point

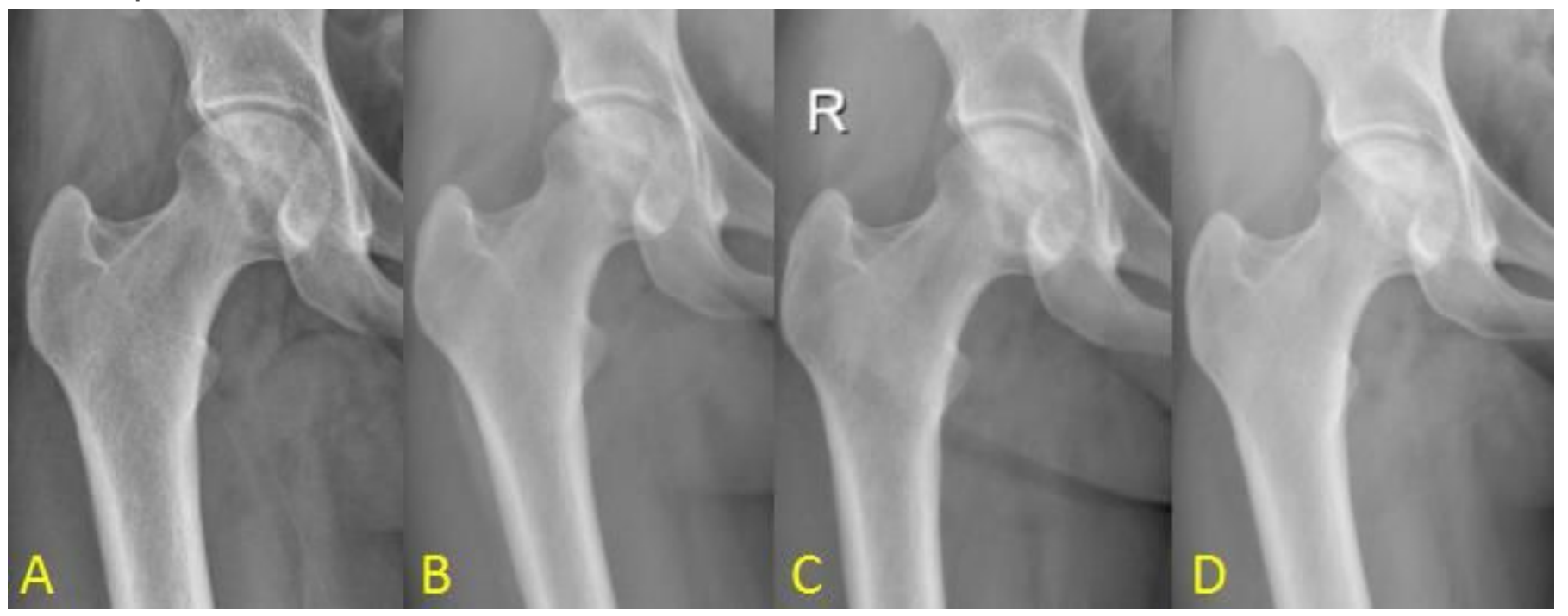

Figure 7

A 25-year-old woman who had steroid-associated osteonecrosis in the left femoral head. (A) Her first-visit medial space ratio was 5.93. (B) After conservative treatments with TCM for 1 year, no obvious collapse of the femoral head could be observed. (C) After two-year TCM treatment, hip symptoms of the patient 
were improved, and the ONFH was being repaired. (D) After 5-year TCM treatment, her hip symptoms basically disappeared, and the necrotic area significantly decreased.

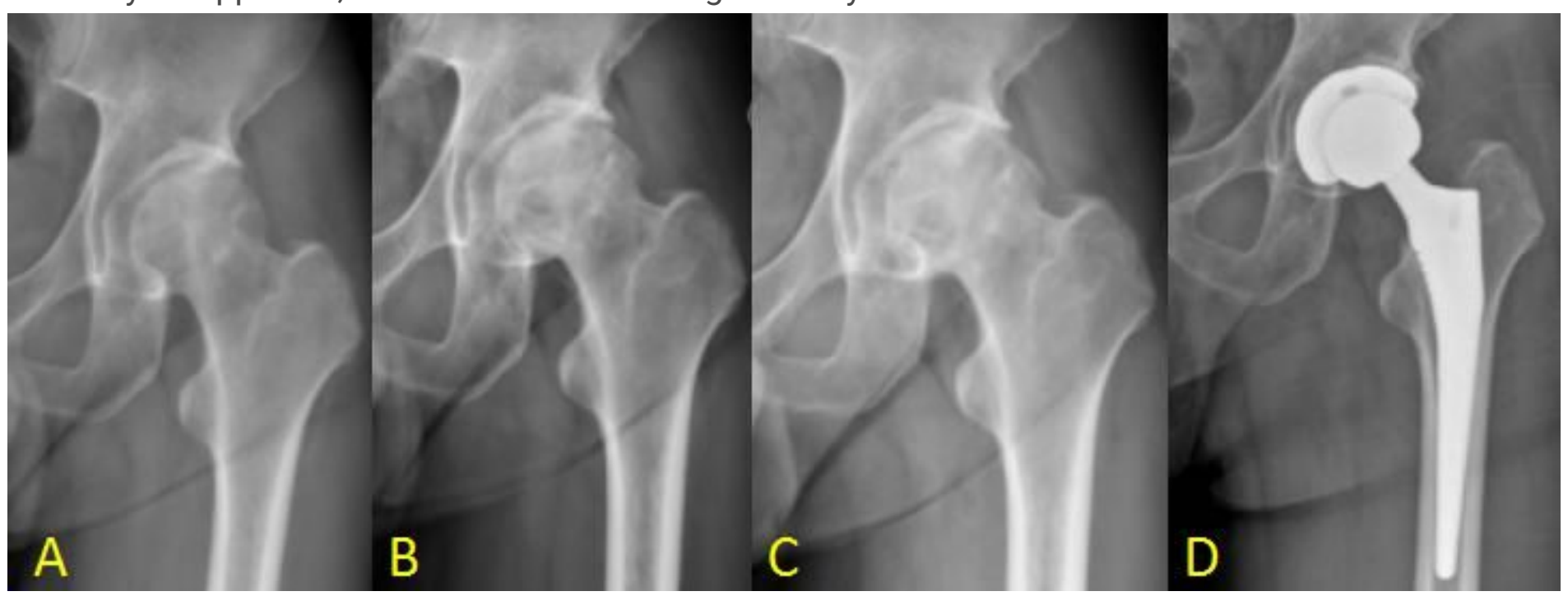

\section{Figure 8}

Radiographs of a 43-year-old man who had alcoholic necrosis in the left femoral head. (A) His first-visit medial space ratio was 3.95. (B) Mild collapse of the femoral head after one-year TCM treatment. (C) After 2-year TCM treatment, collapse of the bearing surface became worse and the articular surface of the femoral head was rough. (D) The patient underwent a total hip replacement. Experimental research results
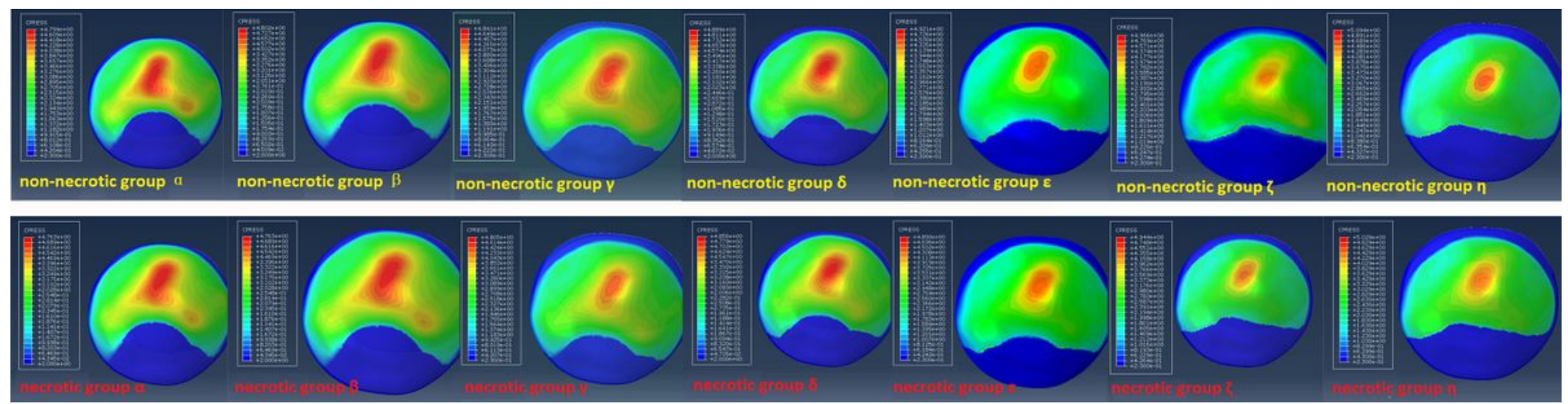

Figure 9

CPRESS stress distribution of femoral head cartilage in each model group 
Femoral head cartilage

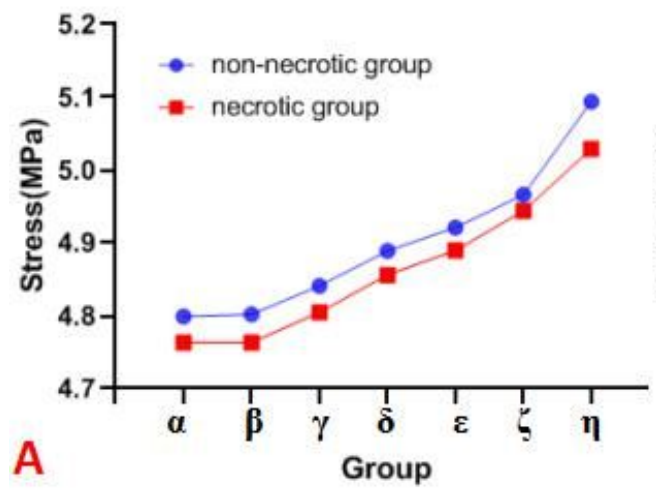

Femoral head cortical bone

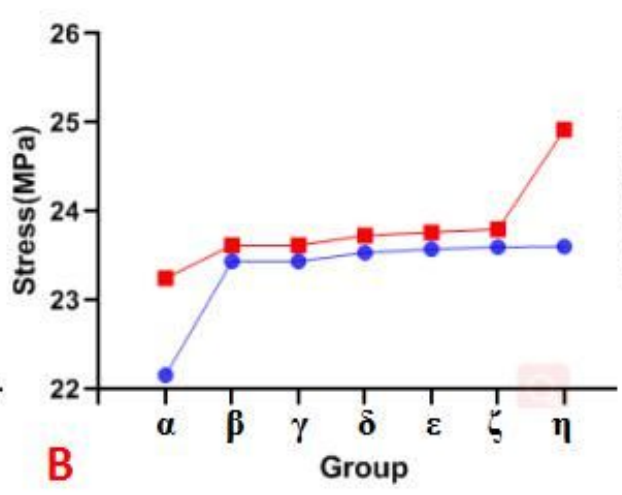

$\mathrm{N}$-unit area

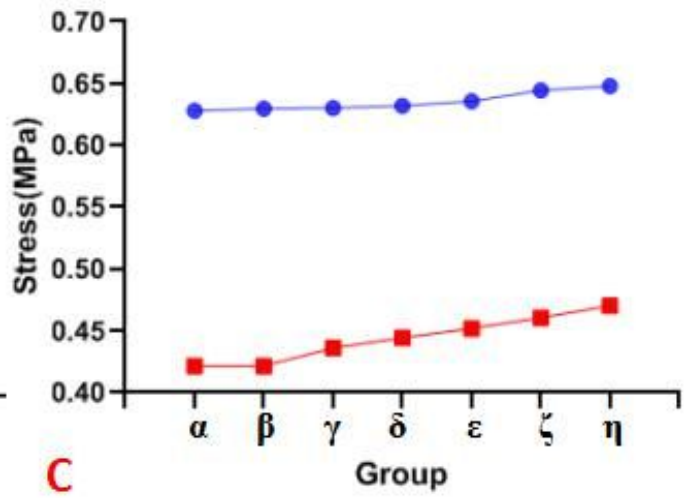

\section{Figure 10}

Maximum VM stress charts. (A) Trends of maximum stress of femoral head cartilage in each model as a function of medial space ratio. (B) Trends of maximum stress in the cortical bone of the femoral head in each model group. (C) Trends of maximum stress of the $\mathrm{N}$-unit area in each model as a function of medial space ratio.
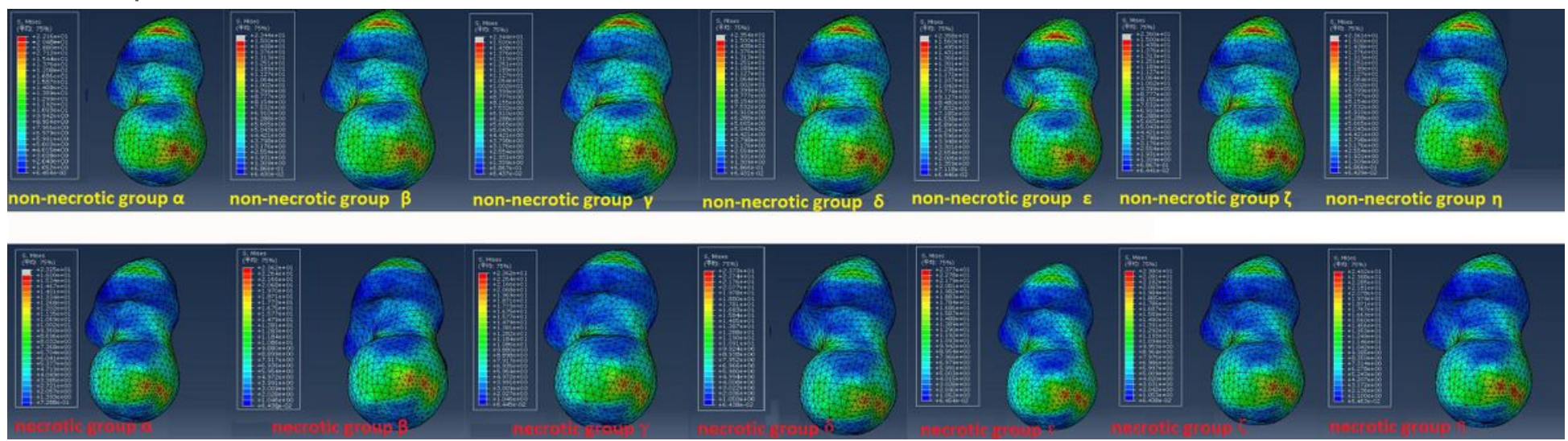

\section{Figure 11}

Distribution of the maximum VM stress of the cortical bone of the femoral head (subchondral bone) in the necrosis group and the non-necrosis group
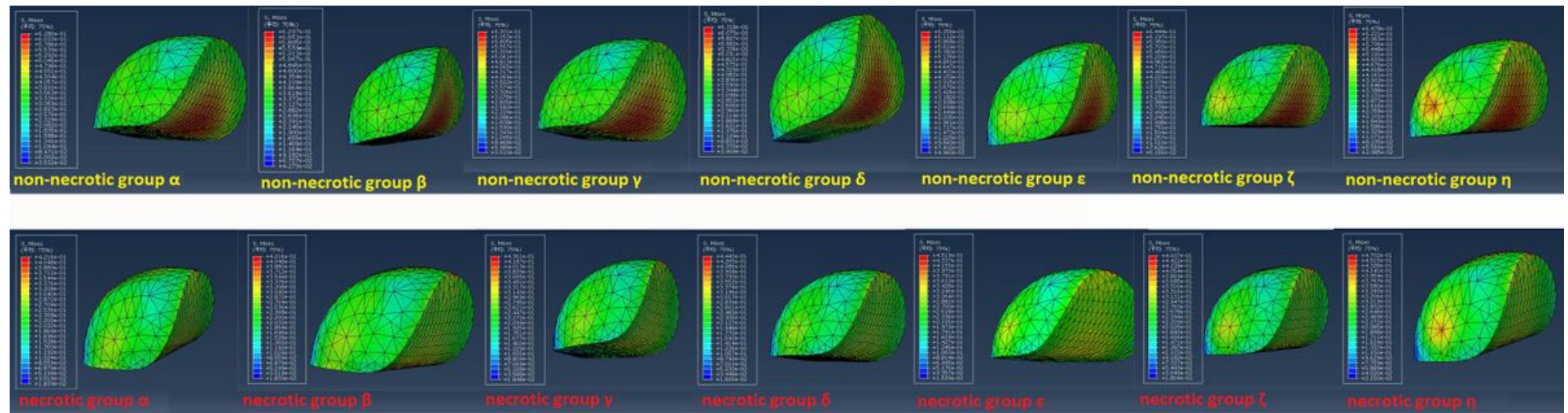

Figure 12 
Distribution of the maximum VM stress in the N-unit area in each model group 